\title{
La adquisición de representaciones menta- les en el niño: resultados de un estudio de caso $^{l}$
}

\author{
IGNACIO MORENO-TORRES \\ SANTIAGO TORRES \\ Universidad de Málaga \\ RAFAEL SANTANA \\ Universidad de Las Palmas de Gran Canaria
}

\section{SOBRE EL ORIGEN DE LAS REPRESENTACIONES MENTALES}

La gran mayoría de los expertos en ciencias cognitivas coinciden en que las representaciones mentales suponen el contenido de la cognición en el hablante adulto (Clapin, 2002; Fodor, 1987). Si el hablante adulto cuenta con tales representaciones, cabe preguntarse si dispone de ellas desde que nace, o, en caso contrario, de qué forma las adquiere. Limitándonos a las representaciones lingüísticas (fonológicas, léxicas, sintácticas), parece claro que al nacer, o bien el niño carece de ellas o bien no están disponibles. Baste pensar que los fonemas aparecen cuando se aprende la lengua materna (Trubetzkoy, 1939; Vihman, 1996), esto es a partir del año. Por ello no puede tener representaciones fonológicas ni léxicas equivalentes a las del adulto. Con respecto a la sintaxis, los innatistas (Chomsky, 1999) defienden que el niño ya dispone de representaciones sintácticas, pues estas se transmitirían genéticamente. Ahora bien, incluso si fuera este el caso, al nacer no las podría usar, ya sea porque se activan al llegar a un grado de maduración determinado, porque se precise un input activador que aún no ha recibido, o por ambos motivos (Locke, 1997).

Dado que al nacer el niño tiene una mínima o nula capacidad de representación, pero que al cabo de pocos años logra manipular complejísimas

1 Este trabajo ha sido elaborado como parte del proyecto de investigación "Desarrollo prelingüístico del niño sordo profundo con implante coclear", subvencionado por la Junta de Andalucía ( ${ }^{\circ}$ P07-SEJ-03119). 
representaciones, parece claro que debe venir dotado de unas habilidades excepcionales para reconocer y producir representaciones mentales, y de una gran capacidad de aprender. Tomasello (2003) agrupa en dos las habilidades básicas innatas del niño: 1) una cognición-social que le permite reconocer la intención comunicativa del otro, y hacer patente la propia (percibir estados mentales del interlocutor, hacer aportaciones cooperativas, etc.), y 2) una cognición-básica que le permite identificar y manipular patrones de muy diverso tipo (auditivos, visuales, táctiles...), ya sean concretos o abstractos. A estas habilidades generales podemos añadir otro requisito obvio para el desarrollo: los interfaces para la entrada y salida de información, que para la lengua oral incluye la audición y el sistema articulatorio. Dotado del soporte biológico, y gracias a los dos tipos de habilidades que acabamos de indicar, el niño recorre en tres o cuatro años (Locke, 1997) el largo camino que a nuestra especie costó recorrer cientos de miles de años. Aunque nuestro objetivo es estudiar cómo es este proceso en un caso atípico, empezaremos por describirlo sucintamente en un caso típico.

\section{I.1. EL DESARROLLO EN EL NIÑO TÍPICO}

Entre otras muchas habilidades, en el primer año de vida, el niño aprende a manipular dos tipos de representaciones que lo preparan para la pronta adquisición del lenguaje: 1) las primeras representaciones de base fonética, manifestadas en las primeras palabras y posiblemente antes en algunas vocalizaciones; y 2) las primeras representaciones semánticas, manifestadas en gestos, juego simbólico y también en las primeras palabras (McCune, 1995). Ahora bien, como ha mostrado la psicología cognitiva, para manipular tales representaciones complejas es preciso realizar toda una serie de procesos cognitivos básicos, y en particular es fundamental la Memoria de Trabajo (MT) (Baddeley, 2007). Nos detendremos brevemente en los diferentes componentes de la MT.

La Memoria de Trabajo (MT) consta de un Ejecutivo Central (EC), que opera como un sistema atencional con dos sistemas esclavos: el Bucle Fonoarticulatorio (BFA), donde se almacena y procesa información verbal durante breve tiempo en estado precategorial, y la Agenda Viso-espacial (AVE), equivalente del BFA pero respecto de los contenidos viso-espaciales. El BFA tiene un papel central en la codificación y almacenamiento temporal de la información fonológica. Por ello, en los términos de este trabajo, tiene un papel central en la adquisición de nuevas representaciones fonológicas. La eficiencia del BFA correlaciona fuertemente con la adquisición del léxico (Gathercole \& Baddeley, 1989). La AVE tiene la función de integrar la información espacial, visual y posiblemente kinestésica en una representación única. Interviene en el lenguaje para manipular representaciones visuo-espaciales, y podría tener un 
papel indirecto en el desarrollo lingüístico. En los últimos años, se ha mostrado evidencia de un cuarto componente, el búfer episódico (BE), que se postula como un espacio mental intermedio entre la MT y la memoria largo plazo (MLP), o sea, nuestra memoria reciente (Baddeley, 2000).

En general se acepta que la MT se desarrolla al mismo tiempo que el niño empieza a manipular sus primeras representaciones. Dicho de otra forma, durante los primeros años de vida se crean las estructuras neuronales que dan soporte a la MT (Rothbart \& Posner, 2001). Una prueba de esta relación es que en condiciones de aislamiento lingüístico los progresos son limitados tanto en tareas dependientes de la MT como en lenguaje (Alegría, 1996).

En resumen, en sus primeros años de vida el niño no sólo aprende a manipular representaciones mentales complejas (como las fonológicas y semánticas), sino que también debe desarrollar las habilidades cognitivas básicas (y en particular las ligadas a la MT) que le permiten operar con tales representaciones complejas. Es por ello, que examinamos ambos aspectos en paralelo.

\section{I.2. SORDERA E IMPLANTE COCLEAR}

En el desarrollo del niño sordo con implante coclear (IC), podemos distinguir dos fases separadas por la colocación del IC. Durante la primera fase (pre-IC) la carencia auditiva será un obstáculo para desarrollar habilidades perceptivas comparables a las del oyente (Jusczyk, 2000). Además, la falta de input hace que la producción no siga el patrón de desarrollo típico (Vihman, 1996). Aunque inicialmente (hasta los 6-8 meses) emite sonidos similares a los del niño típico, pasado este periodo, y debido a la falta de feedback, cesa de emitir vocalizaciones. Naturalmente, si no se toman medidas compensatorias, su léxico oral será pobre o incluso inexistente, en cuyo caso tampoco podría adquirir la gramática. La investigación con niños sordos sin IC permitió mostrar que estos no sólo tenían un claro retraso lingüístico, sino que además mostraban bajo rendimiento en tareas cognitivas básicas dependientes del Bucle Fonoarticulatorio (Alegría, 1996, para una revisión).

La colocación del IC temprano (antes de los 2 años) hace posible que el patrón de desarrollo del sordo implantado se acerque mucho más al del oyente que al del sordo no implantado. Con el IC se producen prontas mejoras en percepción (Blamey, Sarant, Paatsch, et al., 2001; Geers, Brenner, \& Davidson, 2003), con lo que aumenta el input recibido. La mejora en percepción se manifiesta en producción de vocalizaciones desde las pocas semanas o meses (Ertmer \& Mellon, 2001); a más largo plazo el IC hace posible un notable desarrollo léxico y gramatical (LeNormand, 2004; Szagun, 2004). No obstante, estos mismos trabajos muestran que el desarrollo lingüístico no es aún equivalente al del oyente. 
Una pregunta esencial en este contexto es la siguiente: ¿hasta qué punto el IC hace posible un desarrollo cognitivo básico comparable al de los oyentes? En una larga serie de estudios, el grupo de la Universidad de Indiana encabezado por D. Pisoni ha explorado esta cuestión (para una revisión véase Pisoni, Conway, Kronenberger, et al., 2007). Las conclusiones preliminares de esta línea de investigación son que, en diferente grado, y con importantes diferencias intra-grupo, los niños implantados pueden manifestar dos tipos de problemas cognitivos: de procesamiento automático y de procesamiento voluntario. Los problemas de procesamiento automático tendrían su origen en uno de los componentes de la MT, el Bucle Fonoarticulatorio, y en particular en la velocidad de repaso (que se manifiesta en tareas como la repetición de dígitos directos o la velocidad de articulación). Nótese que a nivel lingüístico, esta dificultad debe tener consecuencias directas, al menos en la adquisición del léxico (Gathercore \& Baddeley, 1989) e indirectas, en todo el lenguaje. Con respecto a los problemas de procesamiento voluntario, estos podrían tener su origen, no ya en habilidades directamente dependientes de la audición (como el BFA), sino en habilidades generales, y en concreto en las funciones ejecutivas. Según Pisoni, las funciones ejecutivas se desarrollan en el niño en paralelo con otros aspectos del desarrollo neuronal, especialmente los circuitos del lóbulo frontal, que están densamente conectados con otras regiones del cerebro. Esto es, podría ser que la falta de audición afecte indirectamente a estas habilidades (dado que las interconexiones no pueden darse sin audición). Este déficit tendría impacto, según Pisoni, en un variado conjunto de habilidades y comportamientos: atención e inhibición, memoria y aprendizaje, procesamiento visuoespacial $\mathrm{y}$ funciones senso-motoras.

En este contexto quedan varias preguntas no resueltas. No queda claro por qué estas dificultades en conjunto (de procesamiento automático y procesamiento voluntario) se manifiestan de manera aparentemente arbitraria: algunos niños parecen desarrollarse como los oyentes pero otros muestran dificultades como las que acabamos de indicar. Tampoco queda claro si a más largo plazo los niveles de lenguaje de estos niños serán satisfactorios o si mostrarán déficits localizados (Szagun, 2004).

Por último, el panorama que acabamos de presentar puede empeorar cuando el niño sufre además de la sordera otro déficit asociado, situación que se da en el 30\% de los niños en la actualidad (Edwards, 2007). Sobre el caso particular que nos ocupa, sordera y trastorno por déficit de atención (TDA), diversos estudios coinciden en que el IC resulta beneficioso (Pundir, Nagarkar, \& Panda, 2007; Vlahovic \& Sindija, 2004; Waltzman, Scalchunes, \& Cohen, 2000), pero apenas se detalla el proceso de adquisición. Existe por tanto una importante laguna sobre este ámbito. 


\section{I.3. El TRASTORNo Por DÉfiCIT DE AtENCIÓN (TDA)}

El TDA es el trastorno psiquiátrico más frecuente en la edad escolar, afectando a un promedio del 7\%, aunque el rango de variabilidad va desde el $2 \%$ al 14\% (Scahill \& Schwab-Stone, 2000), dependiendo de los criterios que se adopten para su diagnóstico. Es de origen biológico y de transmisión genética en el $75 \%$ de los casos, siendo más frecuente en varones que en mujeres.

En general, los niños con TDA adquieren tarde el lenguaje (Ygual, 2003, para una revisión) y cometen errores en todos los dominios lingüísticos (fonología, morfosintaxis y semántica). Sin embargo, dado que sus mayores dificultades aparecen en pragmática y que la mayoría de estos niños llegan a dominar el lenguaje, se ha sugerido que sus errores podrían reflejar problemas de actuación más que de competencia (Doyle, 2006; Purvis \& Tannock, 1997). Este hecho puede estar relacionado con el tipo de déficit cognitivo subyacente, como veremos a continuación.

El TDA supone el funcionamiento atípico de las funciones ejecutivas (Doyle, 2006; Purvis \& Tannock, 1997) debido a una menor actividad en los circuitos neuronales que controlan la atención localizados en las áreas prefrontales de la corteza cerebral. Según Barkley (1997) el origen del problema es un fallo en la capacidad de inhibición de la conducta, el primer acto auto-regulatorio, que incide de forma negativa en cuatro funciones neuropsicológicas: a) Memoria de Trabajo; b) Autorregulación de la motivación y el afecto; c) Internalización del lenguaje; y d) Procesos de análisis y síntesis. Pocos estudios han examinado la correlación entre diferentes componentes de la MT y el TDA. En parte ello podría deberse a que los problemas ejecutivos hacen muy difícil evaluar el rendimiento de tareas cognitivas especializadas. Por ejemplo, la repetición de dígitos permite evaluar la eficiencia del BFA, pero presupone un Ejecutivo Central eficiente. Si falla este último, nunca sabremos quién causa los errores al realizar esa tarea (el EC o el BFA). En este sentido, trabajos longitudinales detallados como el presente podrían aportar datos de gran interés, pues la observación continuada facilita la identificación de la naturaleza de los errores. En general, los errores debidos a funciones ejecutivas son más variables, pues dependen de múltiples factores. Por el contrario, los errores en automatismos como el BFA son más estables.

Actualmente diversos estudios científicos indican que el tratamiento con fármacos estimulantes tiene indudables ventajas en el TDA, y es más eficaz que la terapia conductual (Ygual, 2003, para una revisión). Uno de estos fármacos es el metilfenidato, que es un estimulante que aumenta la acción de la dopamina en las áreas prefrontales actuando como inhibidor de su recaptación por la neurona emisora. Este aumento de dopamina en las áreas corticales mejora el control de la inhibición y disminuye el ruido de fondo con el consiguiente aumento de la concentración. 


\section{I.4. OBJETIVOS DEL PRESENTE TRABAJO}

A modo de resumen de este apartado podríamos decir que el desarrollo lingüístico consiste, desde el punto de vista cognitivo, en el desarrollo de la habilidad de manipular representaciones mentales para la interacción social. Lo que la investigación en psicología cognitiva ha mostrado, y en particular las investigaciones de Baddeley y colegas, es que tales habilidades de representación lingüística dependen de otras habilidades subyacentes que se desarrollan en los primeros años de vida.

De gran interés para poner a prueba este esquema evolutivo es el estudio longitudinal de situaciones atípicas en las que se combinan diferentes patologías. Ese es el objetivo fundamental del presente trabajo, en el que examinamos, en una niña nacida sorda y a la que se le diagnosticó TDA a los 5 años de edad: 1) la secuencia de aparición de representaciones mentales fonéticas y semánticas; 2) algunas de sus habilidades cognitivas básicas en diferentes momentos. Además de describir la aparición de estas habilidades, intentaremos mostrar la relación entre su lenguaje y la cognición básica.

\section{MÉTODO}

\section{II.1. Participante}

El estudio se centra en $B$, niña con sordera congénita, neurosensorial, profunda y prelocutiva, detectada a los 12 meses de edad. B tiene una deficiencia asociada a la sordera, Trastorno por Déficit de Atención (TDA). Aunque los primeros síntomas claros del TDA se observan a partir de los 3 años, el origen biogenético del TDA, unido al desarrollo diferencial de este caso, permite hipotetizar que estaba presente desde el principio. Las manifestaciones del TDA fueron confirmándose progresivamente. A los 5 años (tras 3 años y medio de uso de IC), inició un tratamiento con Metilfenidato (5 mg. diarios) para el TDA.

Entre los 14 y los 17 meses B utilizó audífonos retroauriculares bilaterales. A los 17 meses recibió un IC, que estuvo operativo al mes siguiente. La mejora en percepción gracias al IC, -medida con la prueba de percepción LittlEars-, fue buena, estando en la media de los niños implantados a su edad (datos del Centro implantador y de un estudio en curso de los autores). B siguió desde los 15 meses el modelo de intervención MOC (modelo oral complementado) (Torres y Ruiz, 1996). El MOC es un programa de intervención estructurado para niños sordos profundos. Entre los elementos clave de este modelo están: 1) el uso temprano de LPC (La Palabra Complementada) como sistema aumentativo de comunicación, que permite compensar vía visual el input oral que se pierde 
por vía auditiva; 2) el entrenamiento cognitivo; 3 ) la participación activa de la familia en el proceso de intervención, que incluye el uso sistemático de LPC en el contexto familiar (cfr. www.uma.es/moc)

\section{II.2. MATERIAL}

Para la recogida de datos se han empleado 155 grabaciones de vídeo de 30 minutos cada una. Las dos primeras grabaciones se hicieron antes de activar el IC. Las 152 restantes se reparten de la siguiente forma: en los primeros dos años, una grabación semanal (alternando entre la casa familiar y el laboratorio). Desde el inicio del tercer año, y hasta que comenzó el tratamiento farmacológico, las grabaciones fueron bisemanales. Una vez comenzado el tratamiento se realizaron 12 grabaciones ( 2 por semana), también alternando casa y laboratorio. Las grabaciones fueron codificadas según la norma CHAT (MacWhinney, 2000) para la posterior extracción de datos. En diversos momentos, se realizaron pruebas estandarizadas, que permitían la comparación de B con niños de desarrollo típico. Para el periodo prelingüístico fueron estas: las escalas McCarthy de aptitudes y psicomotricidad para niños (desarrollo cognitivo y motor) y el cuestionario LittlEars (percepción).

\section{II.3. CODIFICACIÓN}

Dado que los datos corresponden a un periodo largo en el cual las habilidades comunicativas de la niña varían notablemente, se emplearon diferentes criterios de codificación.

En el periodo prelingüístico se codificaron diversos tipos de manifestaciones prelingüísticas y/o comunicativas, que son indicio del desarrollo de representaciones fonéticas y semánticas. Para estudiar las primeras representaciones fonéticas codificamos dos categorías de datos: vocalizaciones y primeras palabras. Para conocer sus representaciones semánticas codificamos las primeras palabras, sus gestos y las escenas de juego simbólico:

a) Desarrollo vocal (vocalizaciones): Se codificaron las vocalizaciones según una versión reducida de la escala SAEVD-R (Nathani, Ertmer \& Stark, 2006), prestando especial atención a la diferencia entre vocalizaciones que contienen al menos una sílaba consonante-vocal frente a las formadas sólo por vocales. No se anotaron las vocalizaciones no comparables con los sonidos adultos (gruñidos, sonidos aspirados o nasales sin vocales, etc.).

b) Primeras palabras. Se anotaron como palabras las producciones en las que se reconocía el patrón fonético adulto, teniendo en cuenta la 
estructura prosódica y fonológica. Semánticamente, se distinguieron las palabras contextuales de las referenciales (McCune \& Vihman, 2001): una palabra es referencial si el niño asocia una misma forma fonética con al menos dos referentes diferentes o con un mismo referente en dos contextos diferentes; una palabra es contextual si no se cumplen las condiciones anteriores.

c) Juego simbólico: Cada escena de juego fue clasificada según la escala de McCune (1995), que distingue 5 niveles de juego simbólico.

d) Gestos: Siguiendo a Butcher y Golding-Meadow (2000), distinguimos 4 tipos de gestos: Deícticos (como SEÑALAR), convencionales (como SÍ, NO, etc.), icónicos (como los gestos que representan diferentes animales como TORO o acciones como SALTAR), y rituales (como ADIÓS, BRAVO, etc.).

En cuanto al periodo lingüístico se codificaron los siguientes aspectos:

a) Errores fonológicos: se anotaron los errores definidos en términos de procesos fonológicos (Ingram, 1981) estructurales, sistémicos o de asimilación.

b) Errores léxicos y gramaticales: se anotaron los errores producidos por sustitución u omisión de una palabra léxica o de un rasgo o palabra gramatical.

c) Errores pragmáticos: las respuestas de B a preguntas directas fueron clasificadas bien como cooperativas o como no-cooperativas (Grice, 1975).

\section{RESULTADOS}

Agrupamos los datos en tres periodos:

1) Periodo prelingüístico: 2 sesiones previas al IC más el primer año de uso del IC.

2) Periodo lingüístico: desde $2^{\circ}$ año de uso del IC hasta el tratamiento farmacológico para el TDA.

3) Periodo con tratamiento farmacológico para el TDA. 


\section{III.1. PERIODO PRELINGÜÍSTICO}

\section{III.1.1. ARTICULACIÓN}

La tabla 1 muestra el número medio de vocalizaciones por sesión en cuatro trimestres, y la proporción de éstas que incluyen una consonante. Nótese que el impacto del IC se nota ya en el primer trimestre, pues antes del mismo la niña no produce sonido alguno. El descenso en el último trimestre indica que aparecen las primeras palabras. Con respecto al contenido de las vocalizaciones, en general se considera un hito en la progresión del niño que estas últimas representen el 20\% (Oller, 2006). Como puede verse, este hito apenas se alcanza en casa, pero sí en logopedia.

\begin{tabular}{|l|c|c|c|c|}
\hline & \multicolumn{2}{|c|}{ Casa } & \multicolumn{2}{c|}{ Logopedia } \\
\hline $\begin{array}{l}\text { Edad } \\
\text { auditiva }\end{array}$ & Media por sesión & \% con consonante & Media por sesión & \% con consonante \\
\hline Pre-CI & 0,0 & & 0,0 & \\
\hline $1-3$ & 26,3 & $9 \%$ & 19,3 & $5 \%$ \\
\hline $4-6$ & 55,7 & $12 \%$ & 89,0 & $7 \%$ \\
\hline $7-9$ & 92,0 & $16 \%$ & 56,0 & $23 \%$ \\
\hline $10-12$ & 26,7 & $18 \%$ & 68,3 & $30 \%$ \\
\hline
\end{tabular}

Tabla 1. Vocalizaciones

\section{III.1.2. JUEGO SIMBÓLICO}

Cuantitativamente el juego simbólico (JS) de B fue escaso en este periodo; además, la duración en la mayoría de las escenas fue muy breve. Como puede verse en la tabla 2, las primeras escenas de JS (nivel 1) aparecen justo tras la activación del IC. En las dos sesiones pre-IC no aparece ninguna escena. A pesar de que la niña tuvo ocasiones para ello, no empleó los objetos que se le dieron para hacer JS. Nótese que cada nuevo nivel de JS aparece antes en logopedia que en casa. Ello puede estar relacionado con la estimulación recibida en las sesiones. El hecho de que no aparezcan escenas de nivel 5 pudo deberse también a que no se dieron las condiciones adecuadas. Pero incluso obviando ese hecho, parece claro que la calidad del JS de B es menor que el observado en otros niños sordos (Yoshinaga-Itano y Snyder, 1999) y oyentes (McCune, 1995) de su edad. 


\begin{tabular}{|c|c|c|}
\hline Niveles de JS & Casa & Logopedia \\
\hline 1 & 18 & 18 \\
\hline 2 & 23 & 20 \\
\hline 3 & 25 & 21 \\
\hline 4 & - & 28 \\
\hline 5 & - & - \\
\hline
\end{tabular}

Tabla 2. Edad cronológica (en meses) a la que alcanza cada nivel de Juego simbólico

\section{III.1.3. GeSTOS NATURALES}

Con respecto a los gestos naturales, nos interesan dos grupos, los deícticos y los icónicos. Los gestos deícticos son indicios de la cognición-social en la medida en que su uso exige valorar la intención comunicativa del otro (Tomasello, 2003). Como muestra la tabla 3, B produjo un importante número de gestos deícticos ya desde antes de recibir el IC. Nótese que el número de gestos deícticos en casa es idéntico pre-IC y tras 10-12 meses de uso del IC. Este dato apunta a que ya desde antes de colocar el IC, B muestra signos de una comunicación intencionada y social, en el sentido expuesto por Tomasello.

\begin{tabular}{|c|c|c|c|c|}
\hline & \multicolumn{2}{|c|}{ Casa } & \multicolumn{2}{c|}{ Logopedia } \\
\hline EAITipo de gesto & Deícticos & Icónicos & Deícticos & Icónicos \\
\hline Pre & 16,0 & 3,0 & 6,0 & 0,0 \\
\hline $1-3$ & 11,7 & 1,0 & 14,0 & 3,5 \\
\hline $4-6$ & 8,3 & 8,3 & 13,3 & 5,0 \\
\hline $7-9$ & 16,0 & 7,3 & 17,0 & 7,2 \\
\hline $10-12$ & 19,3 & 5,0 & 28,2 & 4,5 \\
\hline
\end{tabular}

Tabla 3. Gestos deícticos e icónicos por sesión

Con respecto a los gestos icónicos, estos reflejan la capacidad de hacer representaciones conceptuales (semánticas). Su número estable a lo largo del tiempo sugiere que no tiene necesidad o no sabe usar estos gestos para transmitir información conceptual. 


\section{III.1.4. Lenguaje: Primeras PAlabras}

El gráfico 1 muestra el número acumulado de palabras diferentes producidas por B mes a mes.

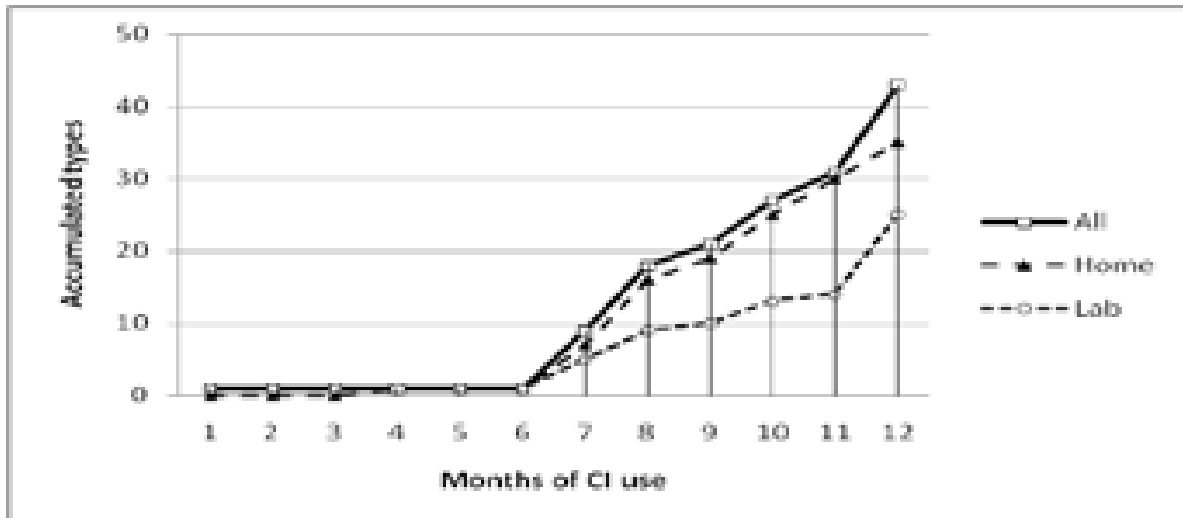

Gráfico 1. Types acumulados durante el primer año de uso del IC

Al final del primer año con IC, B ha producido un total de 43 palabras diferentes de manera espontánea. Todas sus palabras son contextuales (no hay referenciales). Las palabras más frecuentes son en general parte de actos rituales (como los "ahí", "ahítva" o "bien" con que celebra los ejercicios a los que está expuesta frecuentemente), o denominaciones dirigidas por el adulto. Así pues, los datos apuntan a que aún no se ha producido una generalización en el uso de cada etiqueta fonética a diferentes referentes, lo que podría indicar un retraso en la categorización.

\section{III.2. PERIODO LINGÜÍSTICO}

\section{III.2.1. HABILIDADES COGNITIVAS BÁSICAS}

Durante todo este periodo se observan dificultades cognitivas variadas. En primer lugar, mostró dificultades en tareas lógicas básicas como relacionar y/o comparar objetos complementarios, incluir y excluir objetos en base a adición o substracción de rasgos, aplicar principios de pertenencia a campo semántico, etc. También mostró dificultades de acceso léxico, como por ejemplo la evocación de palabras ante una etiqueta de campo semántico. En estos ejercicios se comportaba como un caso de anomia, lo que contrasta con los buenos resultados obtenidos en la evaluación de conocimiento léxico por designación (Peabody y K-bit). Otros ejemplos típicos fueron sus dificultades con los colores y sus frecuentes parafasias. 
B también tuvo muchas dificultades en resolución de tareas basadas en procesamiento sucesivo y simultáneo. Estas carencias en procesamiento de la información justificarían un desarrollo morfosintáctico irregular.

\section{III.2.2. LENGUAJE}

\section{i. Fonología e inteligibilidad}

En el segundo año de uso de IC, aparecen en B todos los fonemas del español (véase Moreno-Torres y Torres, 2008), gracias a lo cual tras 24 meses de uso de IC pudo producir un importante número de palabras. Sin embargo, su inteligibilidad era todavía baja. En el siguiente año, la progresión se observa especialmente en la inteligibilidad. El gráfico 2 muestra el número medio de errores fonológicos cometidos por B por cada 100 palabras en los 12 meses del tercer año de IC. Como se ve, el número de errores en los dos meses finales del tercer año es apenas una cuarta parte de lo observado en los dos primeros meses de ese mismo año.

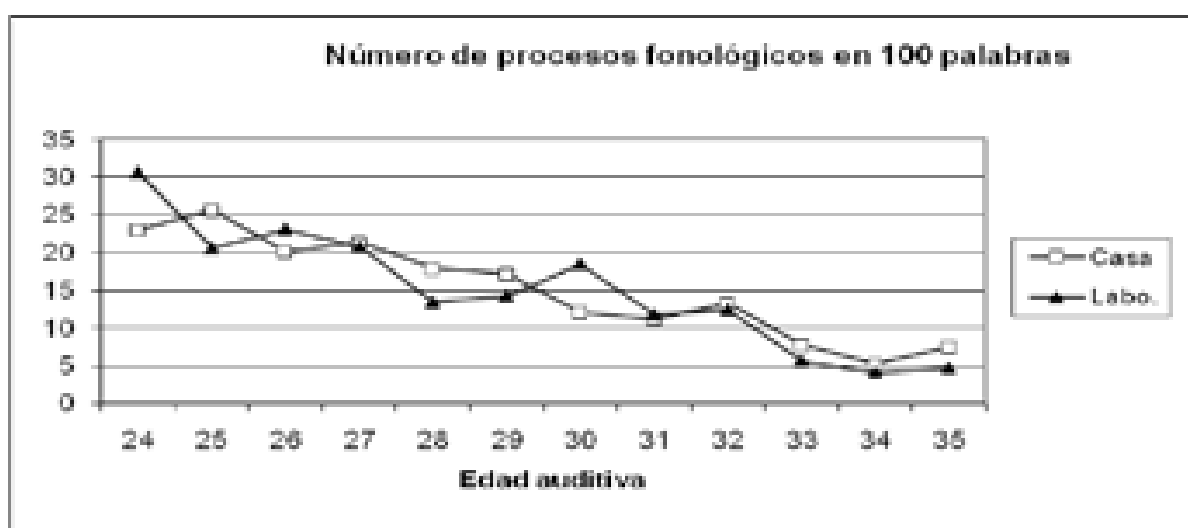

Gráfico 2. Errores fonológicos por mes

Los datos anteriores, obtenidos a partir del corpus, fueron confirmados mediante la Evaluación Fonológica del habla (Bosch, 2004), en la que B obtiene un $100 \%$ de aciertos. A partir de ese momento se da por concluido el desarrollo fonológico al tocar techo en las pruebas diseñadas para oyentes. No obstante, aleatoriamente siguen apareciendo algunos errores fonológicos. 


\section{ii. Léxico}

Justo al comienzo de este periodo (mes 13 de uso del IC), B comienza a usar léxico con valor referencial. Además, se produce un notable aumento en el número de palabras usadas. El avance léxico fue confirmado al final de este periodo con las pruebas de designación léxica de Peabody y K-bit. Ahora bien, hay dos aspectos negativos en este periodo. Por un lado, hay un claro desequilibrio entre la abundancia de nombres empleados y el relativamente escaso uso de verbos y adjetivos (véase Moreno-Torres y Torres, 2008), que sugiere una dificultad para adquirir estos últimos. Por otro lado, aunque B comete pocos errores léxicos (5 por cada 1000 palabras), se detectan dificultades localizadas con los colores y en general con palabras de contenido abstracto como verbos y adjetivos. La tabla 4 muestra los errores léxicos más comunes.

\begin{tabular}{|c|c|c|c|c|c|}
\hline Edad Auditiva & Nombres & Colores & Adjetivos & Verbos & Suma \\
\hline $25-30$ & 5 & 10 & 0 & 4 & 19 \\
\hline $31-36$ & 8 & 10 & 0 & 5 & 23 \\
\hline $37-40$ & 1 & 2 & 5 & 10 & 18 \\
\hline Suma & 14 & 22 & 5 & 19 & 60 \\
\hline
\end{tabular}

Tabla 4. Errores léxicos de B desde los 24 meses de edad auditiva (EA)

Nótese que aunque el número de errores se mantiene, éstos cambian con la edad. Entre los 25-36 meses los errores más comunes aparecen en colores. Luego (37-40 meses), parecen dominar los errores en verbos y adjetivos. Debemos notar que los cambios pueden estar relacionados también con el hecho de que en logopedia se trabajaban aquellos aspectos en los que se detectaban dificultades.

\section{iii. Morfosintaxis}

En el segundo año de uso de IC, aparecen las primeras producciones de dos palabras, y los artículos aparecen en el mismo orden temporal que en los oyentes (Moreno-Torres y Torres, 2008). Estos datos hacían presagiar un rápido desarrollo gramatical. Sin embargo, la progresión es atípica en dos aspectos. Por un lado, aunque los artículos aparecen pronto, B tiende a generalizar al masculino. Este error no suele aparecer en los oyentes (quienes en todo caso tienden a generalizar al femenino). Por otro lado, la progresión de su longitud media de enunciado (LME) es mucho más lenta de lo que cabría esperar una vez que su léxico avanza rápidamente. 
En el tercer año de uso de IC, encontramos una situación similar, con dos aspectos positivos a destacar: 1) desaparece el error de concordancia, y 2) aumenta significativamente la cantidad de estructuras gramaticales y morfemas empleados. Como muestra la tabla 5, el ritmo de aparición de morfemas es casi idéntico en $\mathrm{B}$ y en sus pares oyentes por edad auditiva. Las pocas diferencias se refieren a la preferencia de B por las formas perifrásticas y el retraso del futuro.

\begin{tabular}{|c|c|c|c|}
\hline & $\mathbf{1 8}$ meses & $\mathbf{2 4}$ meses & 30 meses \\
\hline Presente & 2,3, sing & 1 sing, 1,3 plur & \\
\hline Perfecto compuesto & 3 sing & & 1,2 sing \\
\hline Perfecto simple & & & 3 sing \\
\hline Imperfecto & & & 3 sing \\
\hline Futuro & & & 3 sing \\
\hline Subjuntivo & & & \\
\hline Imperativo & 2 sing & & \\
\hline Infinitivo & -ar, -er, -ir & & \\
\hline Gerundio & & -ando, -endo & \\
\hline Perífrasis verbales & & ir+inf / estar+ger & \\
\hline
\end{tabular}

Tabla 5. Edad de aparición de morfemas en B comparada con oyentes*

* Celda gris: morfemas que ya producen oyentes de la misma edad auditiva; en negrita: morfemas que aún no producen los oyentes (Cfr. Serra et al., 2000).

En lo negativo: 1) la LME sigue siendo muy baja, especialmente considerando su progresión léxica y morfológica, y 2) aunque los errores son escasos, se mantienen regularmente y no parecen responder a un patrón en particular: concordancia, elección incorrecta de palabra gramatical y omisiones. La tabla 6 recoge ejemplos de errores lingüísticos de $\mathrm{B}$. Un rasgo común de estos errores es que resulta imposible identificar los roles temáticos de la oraciones:

\begin{tabular}{|c|c|c|}
\hline Edad Aud. & Produce & Comentario \\
\hline $\mathbf{3 8}$ meses & choca un árbol & Omite "contra". Debería decir "chocas contra un árbol" \\
\hline $\mathbf{3 8}$ meses & $\begin{array}{c}\text { me han pegado } \\
\text { con el pelo }\end{array}$ & $\begin{array}{c}\text { Error sintáctico: debería decir "me han pegado en el } \\
\text { pelo" o mejor aún "me han tirado del pelo". }\end{array}$ \\
\hline $\mathbf{3 7}$ meses & $\begin{array}{c}\text { Epi me lo } \\
\text { quiero }\end{array}$ & Error léxico: dice "querer" en lugar de "pedir" \\
\hline
\end{tabular}

Tabla 6. Errores lingüísticos 


\section{iv. Discurso}

La tabla 7 recoge el porcentaje de respuestas cooperativas de $\mathrm{B}$, ante preguntas directas del adulto, en 6 periodos de edad auditiva. A pesar de la relativa mejora con el tiempo, parece que un nivel de respuesta inferior al $30 \%$ es claramente poco cooperativo. Este valor tan bajo concuerda con la valoración subjetiva de las logopedas, que consideraban difícil la interacción comunicativa con $\mathrm{B}$, porque a menudo no entendía las instrucciones o preguntas que se le hacían.

\begin{tabular}{|c|c|c|c|}
\hline EdadAud & Sesiones & Pregs/sesión & \% coop \\
\hline $\mathbf{2 4 - 2 6}$ & 6 & 30,8 & $15,5 \%$ \\
\hline $27-29$ & 6 & 36,0 & $3,3 \%$ \\
\hline $30-32$ & 6 & 61,2 & $27,3 \%$ \\
\hline $33-35$ & 6 & 34,2 & $28,3 \%$ \\
\hline $36-38$ & 5 & 66,2 & $35,5 \%$ \\
\hline $38,5-40$ & 5 & 70,2 & $26,3 \%$ \\
\hline
\end{tabular}

Tabla 7. Respuestas cooperativas

Por otro lado, cabe destacar que B muestra en este periodo una clara tendencia a hablar mediante patrones aprendidos, como canciones y frases hechas. Ya con 5 años, desarrolló la estrategia comunicativa de recurrir a la dramatización y a la polifonía vocal para evitar contar hechos pasados, lo que le permitía evitar el estilo indirecto. O sea, en lugar de decir "la seño le dijo que se fuera”, decía "la seño le dijo: ¡vete!", teatralizando la palabra “vete”. Este mecanismo le permitía resolver, bajo demanda, muchos ejercicios de narración de cuentos. También desarrolló la estrategia de adelantarse en los diálogos y hablar mucho para evitar que el adulto le preguntase. De esta manera, evitando las preguntas directas escapaba a su problema de comprensión del que parecía muy consciente.

\section{III.3. EFECTOS DEL TRATAMIENTO FARMACOLÓGICO PARA EL TDA}

\section{III.3.1. COGNICIÓN}

El efecto del tratamiento con metilfenidato fue inmediato y se mostró en la realización exitosa de tareas cognitivas que antes no podía hacer, $v$. $g r .:$ hizo 
gala de conducta comparativa, ej.: relacionar objetos en base a 1, 2 ó 3 rasgos compartidos (gato-gatito se relacionan por ser de la misma familia, gato-perro se relacionan por ser grandes y machos, etc.) También a la inversa, ej.: teniendo a la vista las etiquetas de sexo-tamaño-familia resolvía problemas de conjuntos llegando a la conclusión de que cuántas más etiquetas había menos individuos cumplían la condición, y viceversa, o sea, pudo formar conceptos. También empezó a hacer gala de buen funcionamiento del búfer episódico (BE) de la MT, así como de habilidad inferencial. Paradójicamente, en la evaluación en pruebas de pseudopalabras su resultado fue peor de lo esperado, especialmente en sílabas poco frecuentes, en las quedó por debajo de la puntuación mínima en oyentes. Estos datos apuntan a que su desarrollo del BFA no es tan positivo como daban a entender su desarrollo fonológico y su inteligibilidad.

\section{III.3.2. LENGUAJE}

\section{i. Léxico y morfosintaxis}

Los datos iniciales obtenidos del corpus indican que el tratamiento tiene un impacto positivo sobre los errores sintácticos, que bajan de manera clara, y en menor medida sobre las omisiones y los errores morfológicos. Sin embargo, se produce un efecto inverso sobre los errores léxicos, que se multiplican por tres. La tabla 8 recoge los tipos de errores más comunes en 10 sesiones previas al tratamiento con metilfenidato (pre-Metil) y en las 12 posteriores (post-Metil). Como se puede ver los errores más comunes son los verbos nuevamente. En contra de lo que cabía esperar se mantienen los errores con los colores, aunque ahora son de tipo anómico y no agnósico (como eran antes del tratamiento con metilfenidato).

\begin{tabular}{|c|c|c|c|c|c|}
\hline & Nombres & Colores & Adjetivos & Verbos & Suma \\
\hline Pre-Metil & 1 & 2 & 5 & 10 & 18 \\
\hline Post-Metil & 13 & 6 & 6 & 33 & 58 \\
\hline
\end{tabular}

Tabla 8. Errores léxicos

La evaluación mediante pruebas estandarizadas confirma estos datos. Su puntuación en la prueba Peabody indica que su desarrollo léxico está apenas por encima de su edad auditiva, esto es, mantiene el retraso con los pares oyentes. Este dato parece contradecir los buenos resultados a los 3 años y medio (tras 2 años de IC), aunque podría estar relacionado con que las dificultades de B son selectivas (se limitan a palabras más abstractas). Con el fin de contrastar 
los datos sobre los errores sintácticos, se procedió a evaluar la comprensión gramatical de B. Para nuestra sorpresa los resultados fueron mucho más pobres de lo que los datos del corpus hacían presagiar.

\section{ii. Discurso}

La tabla 9 recoge el porcentaje de respuestas cooperativas de $\mathrm{B}$, ante preguntas directas del adulto, justo antes de iniciar el tratamiento con metilfenidato, y en los dos meses siguientes. Como se observa, la cantidad de respuestas cooperativas aumenta muy significativamente. Este dato concuerda con la apreciación subjetiva de padres y logopedas sobre la habilidad de la niña para mantener un diálogo. A pesar de este cambio, en este momento no han desaparecido las estrategias comunicativas compensatorias que había desarrollado anteriormente, lo que indica que son patrones de comportamiento y comunicación cuya reversión puede ser más compleja.

\begin{tabular}{|c|c|c|c|}
\hline Toma & Sesiones & Preguntas/sesión & \% respuestas coop. \\
\hline 1 mes antes & 5 & 70,2 & $26,3 \%$ \\
\hline 1 mes después & 6 & 55,5 & $80,9 \%$ \\
\hline 2 meses después & 6 & 90,0 & $74,3 \%$ \\
\hline
\end{tabular}

Tabla 9. Respuestas cooperativas antes y después de tomar metilfenidato

\section{DisCUSIÓN}

El objetivo del presente trabajo era comprobar cómo una niña sorda y con déficit asociado (TDA) iba adquiriendo entre los 18 meses y los 5 años dos tipos de habilidades cognitivas: habilidades básicas ligadas a la Memoria de Trabajo, y representaciones lingüísticas complejas. Junto a ello, pretendíamos comprobar si se observaban relaciones entre ambos tipos de habilidades. Resumimos y valoramos ahora estos datos desde un punto de vista lingüístico y psicolingüístico.

\section{IV.1. EXPLICACIÓN LINGÜÍSTICA}

En términos generales nos encontramos con el siguiente contraste en la progresión prelingüística y lingüística: 1) Considerando el retraso inicial, su progresión linguíística parece satisfactoria en lo relativo a la fonología y morfología; 2) sin embargo, B muestra dificultades de base léxico-semántica 
a lo largo de todo el periodo estudiado. Los datos sobre sintaxis son a priori difíciles de interpretar.

Los avances en fonología y morfología son claros desde el segundo año de uso del IC. Así lo confirmaron los datos del corpus y los de la evaluación estandarizada. Recordemos que tras 24 meses de uso del IC, B ya había producido todos los fonemas, aunque sus niveles de inteligibilidad fueran bajos. Recordemos igualmente, que tras 36 meses de uso del IC, su nivel de aciertos en la prueba estandarizada que realizó fue del $100 \%$. Con respecto a la morfología, B parece seguir el ritmo de sus pares por edad auditiva. Esto es, con un retraso en el tiempo, B sigue los mismos pasos que los oyentes. Es interesante notar que B comete diversos errores de regularización. Estos aparecen primero en la morfología nominal y luego en la verbal. En conjunto estos datos sugieren que la manipulación de representaciones fonológicas no representa un problema grave.

Los avances en representaciones semánticas son menos claros ya desde el primer año de uso del IC. En ese periodo, B mostró estas dificultades: 1) el juego simbólico aparece tardíamente, en escenas breves y que a menudo responden a patrones aprendidos en logopedia; 2) aunque en el primer año de IC aparece un número importante de palabras diferentes, no logra emplearlas con valor referencial. Este último aspecto podría tener su origen en una dificultad pragmática, esto es, en que la niña hubiera aprendido a denominar, pero no fuera capaz de usar el lenguaje funcionalmente. Sin embargo, el hecho de que ya antes de recibir el IC haga abundante uso de gestos comunicativos (deícticos) nos lleva a descartar esta opción. La hipótesis alternativa es que B tiene dificultades para categorizar la realidad externa (ya sea a través del juego o del lenguaje). Estas dificultades se mantienen en el periodo lingüístico, observándose en grupos léxicos que tienen en común el representar información abstracta (colores, adjetivos y verbos) por lo que pueden ser más difíciles de categorizar. Y, en contra de lo que cabía esperar, también se observan tras el tratamiento farmacológico. En suma, la dificultad para manipular representaciones semánticas se mantiene en el tiempo. En el siguiente apartado ofrecemos una posible explicación acerca de por qué no desaparece con el tratamiento farmacológico.

El último ámbito que debemos analizar es el desarrollo gramatical. El análisis de sus errores mostró que los errores son relativamente escasos y no presentan patrón claro. Por ejemplo, en general B emplea bien las preposiciones o los pronombres átonos, pero aleatoriamente comete errores de difícil explicación. Eso sí, se observa que un importante número de errores tiene como consecuencia la imposibilidad de identificar los roles semánticos, resultando en enunciados incomprensibles.

Creemos que estos datos se pueden explicar adecuadamente si adoptamos un enfoque lingüístico cognitivo (Talmy, 2000), según el cual las estructuras 
gramaticales son un reflejo formal del contenido semántico abstracto, esto es, reflejan nuestra forma de conceptualizar la realidad. Desde esta perspectiva, podemos sugerir la siguiente explicación. Al igual que B tiene dificultades para conceptualizar información general (colores, adjetivos, verbos), también tiene dificultades para conceptualizar situaciones complejas (eventos, estados) con varios participantes y diferentes relaciones (dar/quitar, sacar/meter...). Dado que sabemos que B tiene esta dificultad para conceptualizar información abstracta (así se manifiesta en el léxico), es natural que se manifieste también a la hora de intentar describir situaciones cognitivamente complejas.

Por otro lado, los datos de la última evaluación sugieren un posible contraste entre producción y comprensión gramatical. En términos generales su producción parece mejor que su comprensión. Creemos que el contraste producción/comprensión podría ser más aparente que real. En producción el hablante elige las unidades que quiere emplear (lo hacemos cuando hablamos una lengua extranjera). En comprensión debemos procesar lo que otros deciden usar. B se ha mostrado siempre muy hábil para sortear sus dificultades comunicativas, diseñando ingeniosas estrategias y apoyándose en expresiones aprendidas. Por ello es muy posible que a la hora de hablar tenga la tendencia a evitar situaciones difíciles. En resumen, no podemos concluir que haya un contraste producción/comprensión con ventaja hacia la producción.

Para terminar, nótese que la explicación que proponemos para sus dificultades gramaticales, de base semántica, permite limitar a un solo ámbito el origen de las dificultades de B. Creemos que el origen principal de sus dificultades lingüísticas es de origen semántico, y se manifiesta tanto en la conceptualización lexical como en la conceptualización gramatical. Como veremos a continuación, no queremos decir con ello que sus habilidades fonológicas sean iguales a las de sus pares; es sólo que son lo suficientemente buenas como para que, a estas alturas, no tengan efectos adversos aparentes.

\section{IV.2. EXPLICACIÓN PSICOLINGÜÍSTICA}

Aún a sabiendas de la dificultad de la tarea, la gran cantidad de datos obtenidos sobre este caso permiten sugerir una explicación unificada de la evolución de B, y en particular para el patrón que hemos indicado: mejor progreso en fonología-morfología, peor progreso en semántica-gramática. Ese patrón sugiere que la progresión en los componentes de la MT antes citados, sobre todo el BFA y la AVE, es desigual. Analicemos primero los indicios sobre el funcionamiento del BFA.

A pesar del déficit auditivo, el BFA de B ha debido madurar lo suficiente como para que esta logre los avances ya reseñados: rápido desarrollo fonológico, mejoras en inteligibilidad, buena morfología (con ejemplos de regularizaciones). 
Esto es una buena noticia, pues durante décadas se ha apuntado al mal funcionamiento del BFA, y a sus consecuencias más observables, -déficit fonológico y bajo nivel lector-, como las característica distintivas de los sujetos sordos sin IC. Ahora bien, la evaluación final siembra una duda que no podemos obviar: a pesar de las evidentes mejoras, las pruebas psicolingüísticas finas sugieren que su BFA no es tan eficaz como el de los oyentes.

Por otro lado, diversos indicios sugieren que la AVE de B ha podido lastrar sus progresos a lo largo de estos años. Recordemos que la AVE es el sistema donde se procesan las imágenes mentales. Pues bien, dado que el juego simbólico presupone este tipo de operación, es razonable considerar que su pobre JS pre-IC y durante el primer año de uso del IC es una consecuencia de una AVE ineficiente. El retraso en el léxico referencial podría tener el mismo origen (aunque también puede haber incidido aquí un BFA pobre), pues para usar el léxico referencialmente una de las condiciones es haber generalizado las representaciones mentales de objetos. En el periodo lingüístico encontramos más indicios de un pobre desarrollo de la AVE. Comete errores con los colores, y luego con los verbos, todo lo cual sugiere una dificultad para representar conceptos abstractos, tarea en la cual tiene un importante papel la AVE. Sus errores gramaticales aleatorios a partir del tercer año de IC también podrían tener su origen en este componente de la MT. Nótese que la producción de frases es una tarea que se realiza a partir de las imágenes mentales complejas. Como ya apuntábamos antes, si tenía dificultades con las partes (verbos, adjetivos...), también debiera tenerlas con el todo (enunciados). Como el problema es aleatorio, pues en parte depende de los recursos disponibles, se explica que no se observaran patrones de error. En pragmática el problema es similar, aunque es posible que la comprensión sea la causa más inmediata. Si no lograba retener las imágenes simples, tampoco podría retener las imágenes complejas y difícilmente sus respuestas podían ser cooperativas. Por último, el contraste observado, tras el tratamiento para el TDA, entre el aumento de los errores léxicos y la disminución de errores ejecutivos podría explicarse de la siguiente forma: el fármaco aumenta la eficiencia de su MT en general, y del EC en particular, pero no resuelve otros problemas. En particular, si debido a una MT ineficiente el léxico de $\mathrm{B}$ era pobre, una mejora ejecutiva no va a resolver el problema de inmediato. En términos sencillos, si B no ha memorizado y relacionado un buen diccionario, ningún fármaco le va a dar ese diccionario. Lo que sí le proporcionará es la capacidad para procesar información y estar en disposición para alcanzar el mismo diccionario que los demás hablantes oyentes.

En resumen, el impacto de la sordera sobre el BFA y del TDA sobre la AVE podrían explicar a grandes rasgos la progresión de la niña. Pero además, muestran que a pesar de todo el esfuerzo realizado -atención temprana, IC, logopedia intensiva, tratamiento con metilfenidato, etc.-, a día de hoy ni su 
MT ni su lenguaje son equiparables a los del oyente (a pesar de las notables mejoras).

\section{CONCLUSIONES}

El presente análisis ha pretendido ilustrar cómo el desarrollo del lenguaje, entendido como la adquisición de habilidades para manipular representaciones mentales, permite un análisis completo de casos complejos como el aquí examinado. A pesar de tratarse de un estudio de caso, cuyos resultados son por ello difíciles de generalizar, creemos poder extraer algunas conclusiones.

Desde una perspectiva teórica, creemos que estudios que aborden el desarrollo del lenguaje desde el doble punto de vista adoptado aquí, con datos cognitivos básicos y datos lingüísticos, pueden aportar elementos de gran interés sobre debates de fondo como la especificidad o no del lenguaje. En particular, de existir un módulo lingüístico independiente en el cerebro cabría esperar que el desarrollo del lenguaje fuera relativamente independiente de otras habilidades básicas. Los resultados de este estudio sugieren que el lenguaje no es independiente de esas otras habilidades. En efecto, en B el desarrollo lingüístico parece lastrado por una habilidad general (conceptualización semántica) que usamos en diferentes tareas (juego simbólico, gestos, lenguaje...) y que parece ligada a un componente específico de la MT. Los efectos de esta dificultad se manifiestan de diferentes formas y actividades según el momento evolutivo: primero se observa en juego simbólico, luego en la lenta aparición de las primeras palabras, más adelante en los errores léxicos con conceptos abstractos, y finalmente en la gramática. Indirectamente, también afecta a la pragmática. Pero la causa, creemos, es única: es su dificultad para categorizar la realidad. Esta asociación es la que predice la lingüística cognitiva (Talmy, 2000; Langacker, 1987).

Desde una perspectiva aplicada, el presente estudio de caso muestra la necesidad de abordar la intervención logopédica desde una perspectiva global. Esto es, asumiendo que el desarrollo lingüístico depende de habilidades básicas que se adquieren con el tiempo y en recíproca interacción entre lenguaje y cognición. En ese sentido, conviene reflexionar sobre la validez de algunas pruebas de evaluación, realizadas desde una perspectiva muy específica y que, por tanto, podrían no ser capaces de valorar la progresión real. En el presente estudio de caso hemos observado este fenómeno con las pruebas de evaluación léxica e incluso con los datos del corpus. En ocasiones los resultados eran engañosos y sólo un análisis cualitativo permitió descubrir la situación real. 


\section{REFERENCIAS}

ALEGRÍA, J. (1996). "On the origin of phonological representations in deaf people”. En M. Carreiras, I. García-Albea, \& N. Sebastián-Gallés (Eds). Language processing in Spanish. Mahwah, New Jersey: Lawrence Erlbaum Ass., pp. 119-143.

BADDELEY, A. (2000). "The episodic buffer: a new component of working memory?". Trends in Cognitive Sciences, 4(11), 417-423.

BADDELEY, A. D. (2007). Working memory, thought, and action. NY: Oxford University Press.

BARKLEY, R. A. (1997). "Behavioural inhibition, sustained attention, and executive functions: Constructing a unifying theory of ADHD". Psychological Bulletin, 121, 65-94.

BLAMEY, P., SARANT, J., PAATSCH, L., BARRY, J. G., BOW, C. P., WALES, R. J., et al. (2001). "Relationships among speech perception, production, language, hearing loss, and age in children with impaired hearing". Journal of Speech, Language, and Hearing Research, 44, 264-285.

BOSCH, L. (2004). Evaluación fonológica del habla infantil. Barcelona: Masson.

BUTCHER, C., \& GOLDIN-MEADOW, S. (2000). "Gesture and the transition from one- to two-word speech: When hand and mouth come together". In D. McNeill (Ed.), Language and Gesture (pp. 235-257). Cambridge, MA: Cambridge University Press.

CHOMSKY, N. (1999). El Programa Minimalista. Madrid: Alianza Editorial.

CLAPIN, H. (2002). Philosophy of mental representation. Oxford University Press.

DOYLE, A. E. (2006). "Executive functions in attention-deficit/hyperactivity disorder". The Journal of clinical psychiatry, 67, S37-41.

EDWARDS, L. C. (2007). "Children with cochlear implants and complex needs: A review of outcome research and psychological practice". Journal of Deaf Studies and Deaf Education, 12, 258-268.

ERTMER, D. J., \& MELLON, J. A. (2001). "Beginning to talk at 20 months: Early vocal development in a young cochlear implant recipient". Journal of Speech, Language, and Hearing Research, 44, 192-206.

FODOR, J. (1987). Psychosemantics: The Problem of Meaning in the Philosophy of Mind (Psicosemántica. El problema del significado en la filosofía de la mente. Madrid: Tecnos, 1994).

GATHERCOLE, S. E., \& BADDELEY, A. D. (1989). "Development of vocabulary in children and short-term phonological memory". Journal of memory and language, 28, 200-213. 
GEERS, A., BRENNER, C., \& DAVIDSON, L. (2003). "Factors associated with development of speech perception skills in children implanted by age five". Ear and Hearing, 24, 24S-35S.

GRICE, H. P. (1975). "Logic and conversation". En P. Cole, \& J. L. Morgan, (eds.). Syntax and Semantics Vol 3: Speech Acts. Nueva York: Academic Press.

INGRAM, D. (1981). Procedures for the phonological analysis of children's language. Baltimore, Md.: University Park Press.

JUSCZYK, P. (2000). The discovery of spoken language. Cambridge, Mass.: The MIT Press.

LANGACKER, R. (1987). Foundations of Cognitive Grammar. Stanford, CA: Stanford University Press.

LE NORMAND, M. T. (2004). "Evaluation du lexique de production chez des enfants sourds profonds munis d'un implant cochléaire sur un suivi de trois ans ». Rééducation orthophonique, 42, 125-140.

LOCKE, J. L. (1997). “A Theory of Neurolinguistic Development”. Brain \& Language, 58, 265-326.

MacWHINNEY, B. (2000). The CHILDES Project: Tools for Analyzing Talk. Mahwah, NJ : LEA.

McCUNE, L., \& VIHMAN, M. M. (2001). "Early phonetic and lexical development: A productivity approach". Journal of Speech, Language, and Hearing Research, 44, 670-684.

McCUNE, L. (1995). "A normative study of representational play at the transition to language". Developmental Psychology, 31(2), 198-206.

MORENO-TORRES, I., \& TORRES, S. (2008). "From 1-word to 2-words with Cochlear Implant and Cued Speech: A case study". Clinical Linguistics and Phonetics, 22(7), 491-508.

MORENO-TORRES, I., TORRES, S. \& SANTANA, R. (en preparación). El desarrollo prelingüístico y lingüístico del niño sordo con implante coclear.

NATHANI, S., ERTMER, D., \& STARK, R. (2006). "Assessing vocal development in infants and toddlers". Clinical Linguistics \& Phonetics, 20(5), 351-369.

OLLER, D. K. (2006). "Vocal language development in deaf infants: new challenges". En P. Spencer, \& M. Marschark (Eds.), Advances in the spoken language development of deaf and hard of hearing children. (pp. 23-41). NY: Oxford University Press.

PISONI, D. B., CONWAY, C. M., KRONENBERGER, W., HORN, D. L., KARPICKE, J. \& HENNING, S. (2007). "Efficacy and Effectiveness of Cochlear Implants in Deaf Children". Research on Spoken Language Processing, Progress Report, núm. 28. Indiana University. 
PUNDIR, M., NAGARKAR, A. N., \& PANDA N. K. (2007). "Intervention strategies in children with cochlear implants having attention deficit hyperactivity disorder", International Journal of Pediatric Otorhinolaryngology, 71, 985-8.

PURVIS, K. L., \& TANNOCK, R. (1997). "Language abilities in children with attention deficit hyperactivity disorder, reading disabilities and normal control". Journal of Abnormal Child Psychology, 25, 133-144.

ROTHBART, M. K., \& POSNER, M. I. (2001). "Mechanism and variation in the development of attentional networks". En C. A. Nelson, \& M. Luciana, (Eds.), Handbook of developmental cognitive neuroscience. (pp. 353-363). Cambridge, MA: MIT Press.

SCAHILL, L., \& SCHWAB-STONE, M. (2000). Epidemiology of ADHD in school-age children. Child Adolesc Psychiatr Clin N Am, 9, 541-555.

SERRA, M., SERRAT, E., SOLÉ, R, BEL, A., \& APARICI, M. (2000). La adquisición del lenguaje. Barcelona: Ariel.

SZAGUN, G. (2004). "Learning by ear: On the acquisition of case and gender marking by German-speaking children with normal hearing and with cochlear implants". Journal of Child Language, 31, 1-30.

TALMY, L. (2000).Toward a cognitive semantics (2 vol.). Cambridge, MA: MIT Press.

TOMASELLO, M. (2003). Constructing a Language: A Usage-Based Theory of Language Acquisition. Harvard University Press

TORRES, S., \& RUIZ, M. J. (1996). La Palabra Complementada. Madrid: CEPE.

TRUBETZKOY, N. S. (1939). Principios de fonología. Madrid: Cincel (1987).

VIHMAN, M. M. (1996). Phonological development: The origins of child language. Oxford: Blackwell.

VLAHOVIC, S., \& SINDIJA, B. (2004). "The influence of potentially limiting factors on pediatric outcomes following cochlear implantation". International Journal of Pediatric Otorhinolaryngology, 68, 1167-1174.

WALTZMAN, S. B., SCALCHUNES, V., \& COHEN, N. L. (2000). "Performance of multiply handicapped children using cochlear implants". American Journal of Otology, 21, 329-335.

YGUAL, A. (2003). Problemas de lenguaje con estudiantes con déficit atencional. Valencia, Servei de Publicacions, Universitat de Valencia.

YOSHINAGA-ITANO, C., \& SNYDER, L. (1999). "The relationship of language and symbolic play in deaf and hard-of-hearing children". Volta Review, 100, 135-164. 